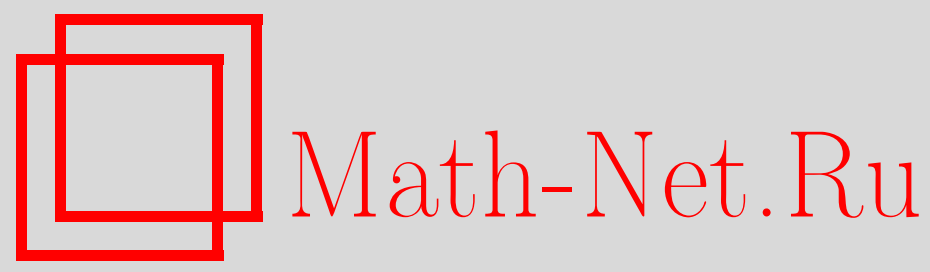

С. В. Хабиров, Приведение инвариантной подмодели газовой динамики к каноническому виду, Матем. заметки, 1999, том 66, выпуск 3, 439-444

DOI: https://doi.org/10.4213/mzm1185

Использование Общероссийского математического портала Math-Net.Ru подразумевает, что вы прочитали и согласны с пользовательским соглашением http://www . mathnet.ru/rus/agreement

Параметры загрузки:

IP: 54.224 .187 .69

26 апреля 2023 г., $17: 13: 22$ 


\section{ПРИВЕДЕНИЕ ИНВАРИАНТНОЙ ПОДМОДЕЛИ ГАЗОВОЙ ДИНАМИКИ К КАНОНИЧЕСКОМУ ВИДУ}

\section{С. В. Хабиров}

В работе рассматривается способ приведения инвариантной подмодели к каноническому виду.

Библиограбия: 4 названия.

Уравнения газовой динамики $D=\partial_{t}+\vec{u} \cdot \nabla$,

$$
\rho D \vec{u}+\nabla p=0, \quad D \rho+\rho \operatorname{div} \vec{u}=0, \quad D p+A(p, \rho) \operatorname{div} \vec{u}=0
$$

допускают 11-ти мерную алгебру Ли $\mathrm{L}_{11}$ преобразований с произвольной функцией $A(\rho, p)$. Для специальных функций $A(\rho, p)$ алгебра расширяется [1]. Для каждой одномерной или двумерной подалгебры можно рассматривать инвариантное решение, если выполнены необходимые условия существования инвариантного решения [2, с. 250]. Все такие подалгебры перечислены с точностью до внутренних автоморфизмов. Для $\mathrm{L}_{11}$ подалгебры сведены в таблицу 6 работы [1], для алгебры Ли $\mathrm{L}_{13}$ политропного газа и алгебры Ли $\mathrm{L}_{14}$ политропного газа с показателем адиабаты $\gamma=5 / 3$ таблищы подалгебр приведены в работах [3] и [4]. Анализ инвариантов подалгебр показьвает, что три инварианта можно выбрать линейными по скоростям $\vec{u}=(u, v, w)$ и независимыми от давления $p$ и плотности $\rho$, один инвариант зависит линейно от давления и независимых переменных, один инвариант зависит линейно и однородно от плотности и независимых переменных. Для того чтобы инварианты можно было выбрать именно такими, необходимо в подалгебрах, содержащих вращение, перейти в цилиндрические координаты, связанные с этим врашением. Остальные инварианты выражаются через независимые переменные $t, x, y, z$.

Инвариантные подмодели приводятся выбором инвариантов к двум каноническим видам систем дифференциальных уравнений. Эволюционньй видполучается, когда время $t$ есть инвариант подалгебры. Система походит на неоднородные уравнения нестационарной плоской или одномерной газодинамики с множителями при градиенте давления. Система смешанного вида походит на неоднородные уравнения стационарной газодинамики с множителями при градиенте давления и получается, когда имеются 3 (или 2 в случае двумерной подалгебры) инварианта из независимьх переменных, из которых определяются пространственные переменные.

Рассматривается способ приведения инвариантной подмодели к каноническому виду.

Работа выполнена при финансовой поддержке Российского фонда фундаментальных исследований, грант № 96-01-01780. 
1. Эволюционная система получается, когда инвариантное решение имеет вид

$$
\vec{u}=B(\vec{v}-\vec{b}), \quad \rho=m R, \quad p=n P+k,
$$

где элементы невырожденной матрищы $B, \vec{b}, m, n, k$ есть функции независимых переменных $t, x, y, z ; \vec{v}, R, P$ есть инвариантные скорости, плотность, давление, зависящие от инвариантов $t, I(x, y, z), J(x, y, z)$.

Подстановка этого вида решения в векторное уравнение системы (1) дает

$$
\vec{v}_{t}+(B(\vec{v}+\vec{b}) \cdot \nabla I) \vec{v}_{I}+(B(\vec{v}+\vec{b}) \cdot \nabla J) \vec{v}_{J}+n m^{-1} R^{-1}\left(P_{I} B^{-1} \cdot \nabla I+P_{J} B^{-1} \nabla J\right) \vec{a}
$$

где величины $B(\vec{v}+\vec{b}) \cdot \nabla I=(\vec{v}+\vec{b}) \cdot B^{T} \nabla I, B(\vec{v}+\vec{b}) \cdot \nabla J=(\vec{v}+\vec{b}) \cdot B^{T} \nabla J, n m^{-1} B^{-1} \nabla I$, $n m^{-1} B^{-1} \nabla J, \vec{a}$ выражаются через инварианты. Выражение для компонент вектора $\vec{a}$ не содержит производных от функций инвариантов.

Дивергенция вычисляется по формуле: $\operatorname{div} \vec{u}=B^{T} \nabla I \cdot \vec{v}_{I}+B^{T} \nabla J \cdot \vec{v}_{J}+\cdots$, где многоточием будут обозначаться слагаемые, не содержащие производных. Вводятся новые инвариантные скорости $u=(\vec{v}+\vec{b}) \cdot B^{T} \nabla I, v=(\vec{v}+\vec{b}) \cdot B^{T} \nabla J, w-$ линейная комбинация скоростей $\vec{v}$, линейно независимая с $u, v$. Тогда первые два уравнения (1) и дивергенщия примут вид

$$
\begin{aligned}
& D_{1} u+R^{-1} n m^{-1}\left(P_{I} \nabla I \cdot \nabla I+P_{J} \nabla I \cdot \nabla J\right)=b_{1}, \\
& D_{1} v+R^{-1} n m^{-1}\left(P_{I} \nabla I \cdot \nabla J+P_{J} \nabla J \cdot \nabla I\right)=b_{2}, \quad \operatorname{div} \vec{u}=u_{I}+v_{J}+\cdots,
\end{aligned}
$$

где $D_{1}=\partial_{t}+\vec{u}_{1} \cdot \nabla_{1}=\partial_{t}+u \partial_{I}+v \partial_{J}$. Таким образом, инвариантная подмодель имеет представление

$$
\begin{aligned}
& D_{1} u+R^{-1}\left(c_{11} P_{I}+c_{12} P_{J}\right)=b_{1}, \\
& D_{1} v+R^{-1}\left(c_{12} P_{I}+c_{22} P_{J}\right)=b_{2}, \\
& D_{1} w+R^{-1}\left(c_{13} P_{I}+c_{23} P_{J}\right)=b_{3}, \\
& D_{1} R+R\left(u_{I}+v_{J}\right)=R b_{4}, \\
& D_{1} P+A(R, P)\left(u_{I}+v_{J}\right)=b_{5} .
\end{aligned}
$$

Систему (2) можно упростить.

Теорема 1. Заменой инвариантов $I, J$ можно привести систему (2) к каноническому әволючионному виду с диагональной матрицей $C=\left(c_{i j}\right), i, j=1,2$.

ДокАЗАтЕльство. Сделаем замену инвариантов $\Phi=\varphi(I, J), \Psi=\psi(I, J)$ с невырожденной матрицей Якоби $M ; u_{1}=\varphi_{I} u+\varphi_{J} v, v_{1}=\psi_{I} u+\psi_{J} v$ или в матричном виде $\vec{u}_{1}=M \vec{u}$. При этом $D_{1}=\partial_{t}+u_{1} \partial_{\Phi}+v_{1} \partial_{\Psi}=\partial_{t}+\vec{u}_{1} \cdot \nabla_{2}=D_{2}, I_{I}+v_{J}=u_{1 \Phi}+v_{1 \Psi}+\cdots$, $\nabla_{1} P=M^{T} \nabla_{2} P$. Первые два уравнения системы $(2)$ в новых переменных примут вид: $D_{1} \vec{u}_{1}+R^{-1} C_{1} \nabla_{2} P=\vec{c}, C_{1}=M C M^{T}$. Остальные не изменяются.

Симметричная матрица $C$ имеет действительные собственные значения

$$
\lambda_{ \pm}=\frac{1}{2}\left(c_{11}+c_{22} \pm c_{12}\left(k^{2}+1\right)^{1 / 2}\right), \quad k=c_{12}^{-1}\left(c_{11}-c_{22}\right)
$$


и действительные ортонормированные собственные векторы

$$
\vec{e}_{+}=(\cos \alpha,-\sin \alpha), \quad \vec{e}_{-}=(\sin \alpha, \cos \alpha), \quad k_{1}=\operatorname{tg} \alpha=\frac{1}{2}\left(k-\left(k^{2}+1\right)^{1 / 2}\right) .
$$

В базисе из собственных векторов с матрищей перехода $N=\left\|\begin{array}{cc}\cos \alpha & \sin \alpha \\ -\sin \alpha & \cos \alpha\end{array}\right\|$ матрища $C_{1}$ становится диагональной: $C_{1} \operatorname{diag}\left(\lambda_{+}, \lambda_{-}\right)=N^{T} C N$. Отсюда следуют уравнения для нахождения функций $\varphi, \psi: \varphi_{I}=\cos \alpha, \varphi_{J}=-\sin \alpha ; \psi_{I}=\sin \alpha, \psi_{J}=\cos \alpha$. Эти системы могут оказаться несовместными.

Для исправления ситуации растягиваются векторы $\lambda \vec{e}_{+}, \mu \vec{e}_{-}$, по которым определяется новая матрица перехода. Тогда диагональная матрица изменится: $C_{1}=\operatorname{diag}\left(\lambda_{+} \mu^{2}\right.$, $\left.\lambda_{-} \lambda^{2}\right)$, а уравнения для нахождения функций $\varphi, \psi$ примут вид:

$$
\varphi_{I}=\lambda \cos \alpha, \quad \varphi_{J}=-\lambda \sin \alpha ; \quad \psi_{I}=\mu \sin \alpha, \quad \psi_{J}=\mu \cos \alpha
$$

Условия совместности систем (3) есть уравнения для определения $\lambda, \mu$ :

$$
\begin{aligned}
\lambda_{J}+k_{1} \lambda_{I} & =\lambda\left(1+k_{1}^{2}\right)^{-1}\left(k_{1} k_{1 J}-k_{1 I}\right), \\
-k_{1} \mu_{J}+\mu_{I} & =\mu\left(1+k_{1}^{2}\right)^{-1}\left(k_{1 J}+k_{1} k_{1 I}\right) .
\end{aligned}
$$

Величины $\lambda$ или $\mu$ могут равняться 1 , если $k_{1} k_{1 J}=k_{1 I}$ или $k_{1 J}+k_{1} k_{1 I}=0$.

В качестве примера рассмотрим одномерную подалгебру 1.21 для политропного газа $A=\gamma p$ из [3]. (В работе [3] приведена 13-мерная алгебра Ли $\mathrm{L}_{13}$, допускаемая уравнениями политропного газа. В ней перечислены все ее подалгебры всех размерностей, которые сведены в таблицу с присвоением каждой алгебре номера. Я рассматриваю одномерную подалгебру с номером 1.21 из таблицы.) В цилиндрической системе координат $x, y=r \cos \theta, z=r \sin \theta, u=U, v=V \cos \theta-W \sin \theta, w=V \sin \theta+W \cos \theta$, оператор одномерной подалгебры такой:

$X_{7}+a\left(X_{11}-X_{13}\right)+b X_{14}=\partial_{\theta}+a\left(x \partial_{x}+r \partial_{r}+U \partial_{u}+V \partial_{V}+W \partial_{w}\right)+(3 a+b) \rho \partial_{\rho}+(5 a+b) p \partial_{p}$.

Инварианты из независимых переменных таковы: $t, s=r e^{-a \theta}, q=x e^{-a \theta}$. Инвариантное решение имеет вид: $U=u^{\prime} e^{a \theta}, V=v^{\prime} e^{a \theta}, W=w^{\prime} e^{a \theta}, \rho=\rho^{\prime} e^{(3 a+b) \theta}$, $p=p^{\prime} e^{(5 a+b) \theta}$. Подстановка в систему $(1)$ приводит к инвариантной подмодели

$$
\begin{aligned}
& D=\partial_{t}+\left(u^{\prime}-a q s^{-1} w^{\prime}\right) \partial_{q}+\left(v^{\prime}-a w^{\prime}\right) \partial_{s}, \\
& D u^{\prime}+\rho^{\prime-1} p_{q}^{\prime}=-a s^{-1} u^{\prime} w^{\prime}, \\
& D v^{\prime}+\rho^{\prime-1} p_{s}^{\prime}=s^{-1} w^{2} \\
& D w^{\prime}-a \rho^{\prime-1}\left(p_{s}^{\prime}+q s^{-1} p_{q}^{\prime}\right)=-a s^{-1} w^{2}-s^{-1} v^{\prime} w^{\prime}-(b+5 a) s^{-1} \rho^{\prime-1} p^{\prime}, \\
& D \rho^{\prime}+\rho^{\prime}\left(u_{q}^{\prime}+v_{s}^{\prime}-a q s^{-1} w_{q}^{\prime}-a w_{s}^{\prime}\right)=-\rho^{\prime} s^{-1}\left(v^{\prime}+w^{\prime}(b+4 a)\right), \\
& D p^{\prime}+\gamma \rho^{\prime}\left(u_{q}^{\prime}+v_{s}^{\prime}-a q s^{-1} w_{q}^{\prime}-a w_{s}^{\prime}\right)=-p^{\prime} s^{-1}\left(\gamma v^{\prime}+w^{\prime}(b+a(\gamma+5))\right) .
\end{aligned}
$$


Замена $u^{\prime}-a q s^{-1} w^{\prime}=u, v^{\prime}-a w^{\prime}=v, p^{\prime}=P, \rho^{\prime}=R, w^{\prime}=w$ приводит к системе (2), где $c_{11}=1+a^{2} q^{2} s^{-2}, c_{12}=a^{2} q s^{-1}, c_{22}=1+a^{2}$,

$$
\begin{aligned}
& b_{1}=2 a w s^{-1}\left(-a+q s^{-1} v\right)+a^{2} q s^{2} w^{2}+a(b+5 a) q s^{-2} P R^{-1} \\
& b_{2}=\left(1+a^{2}\right) s^{-1} w^{2}+a(b+5 a) s^{-1} P R^{-1} \\
& b_{3}=-2 a s^{-1} w^{2}-s^{-1} v w-s^{-1}(b+5 a) P R^{-1} \\
& b_{4}=-s^{-1}(v+(b+5 a) w), \quad b_{5}=-P s^{-1}[\gamma v+(b+(2 \gamma+5) a) w] .
\end{aligned}
$$

Матрица $C$ имеет собственные значения $\lambda_{+}=1, \lambda_{-}=1+a^{2}+a^{2} q^{2} s^{-2}$ и собственные векторы

$$
\vec{e}_{+}=\left(-s\left(q^{2}+s^{2}\right)^{-1 / 2}, q\left(q^{2}+s^{2}\right)^{-1 / 2}\right), \quad \vec{e}_{-}=\left(q\left(q^{2}+s^{2}\right)^{-1 / 2}, s\left(q^{2}+s^{2}\right)^{-1 / 2}\right) .
$$

Замена $I=\varphi(q, s), J=\psi(q, s)$ приводит матрицу $C$ к диагональному виду, если $\psi_{q}=q\left(q^{2}+s^{2}\right)^{-1 / 2}, \psi_{s}=s\left(q^{2}+s^{2}\right)^{-1 / 2} \Rightarrow \psi=\left(q^{2}+s^{2}\right)^{-1 / 2} ; \varphi_{q}=-s\left(q^{2}+s^{2}\right)^{-1 / 2}$, $\varphi_{s}=q\left(q^{2}+s^{2}\right)^{-1 / 2} \Rightarrow$ система не совместна. Для исправления ситуации растягивается вектор $\vec{e}_{+} \rightarrow \lambda \vec{e}_{+}$. Тогда $C_{1}=\operatorname{diag}\left(\lambda_{+} \lambda^{2}, \lambda_{-}\right)$; система (3) имеет вид $\varphi_{q}=$ $-\lambda s\left(q^{2}+s^{2}\right)^{-1 / 2}, \varphi_{s}=\lambda q\left(q^{2}+s^{2}\right)^{-1 / 2} ;$ уравнение (4) $q \lambda_{q}+s \lambda_{s}+\lambda=0$ имеет решение $\lambda=s^{-1} f\left(q s^{-1}\right)$. Выбираем $f(\tau)=-\left(1+\tau^{2}\right)^{1 / 2}$; тогда $q=J \sin I, s=J \cos I(I, J-$ полярные координаты в плоскости $s, q), u=J u_{1} \cos I+v_{1} \sin I, v=-J u_{1} \sin I+v_{1} \cos I$, $D=\partial_{t}+u \partial_{q}+v \partial_{s}=\partial_{t}+u_{1} \partial_{I}+v_{1} \partial_{J}$.

Система (5) примет канонический вид:

$$
\begin{aligned}
D u_{1}+R^{-1} J^{-2} P_{I}= & -2 J^{-1} u_{1} v_{1}-2 a J^{-1} u_{1} w \cos ^{-1} I-J^{-1} w^{2} \operatorname{tg} I, \\
D v_{1}+R^{-1}\left(1+a^{2} \cos ^{-2} I\right) P_{J}= & -J^{-1} w^{2}\left(1+a^{2} \cos ^{-2} I\right)+J u_{1}^{2} \\
& -2 a u_{1} w \sin I \cos ^{-2} I+a(b+5 a) J^{-1} R^{-1} P \cos ^{-2} I, \\
D w-a R^{-1} P_{y} \cos ^{-1} I= & -2 a J^{-1} w^{2} \cos ^{-1} I-w J^{-1}\left(-J u_{1} \operatorname{tg} I+v_{1}\right) \\
& -(b+5 a) J^{-1} R^{-1} P \cos ^{-1} I, \\
D R+R\left(u_{1 I}+v_{1 J}\right)= & -R\left(2 J^{-1} v_{1}-u_{1} \operatorname{tg} I+w J^{-1}(b+5 a) \cos ^{-1} I\right), \\
D P+\gamma P\left(u_{1 I}+v_{1 J}\right)= & -P\left(2 \gamma J^{-1} v_{1}-\gamma u_{1} \operatorname{tg} I\right. \\
& \left.+w J^{-1}(b+(5+2 \gamma) a) \cos ^{-1} I\right) .
\end{aligned}
$$

2. Инвариантная система смешанного типа получается, когда у одномерной подалгебры есть три инварианта $I_{1}, I_{2}, I_{3}$ из независимых переменных системы (1) с неравным нулю якобианом $D\left(I_{1}, I_{2}, I_{3}\right) / D(x, y, x) \neq 0$. Вид инвариантного решения таков: $\vec{u}=B \vec{v}+\vec{b}, \rho=m R, p=n P+k$, где инварианты $\vec{v}, P, R$ зависят от $I_{1}, I_{2}, I_{3}$; элементы невырожденной матрицы $B, \vec{b}, m, n, k$ есть функции независимых переменных.

Подстановка в векторное уравнение системы (1) дает

$$
\vec{v}_{i} D I_{i}+R^{-1} m^{-1} n P_{i} B^{-1} \nabla I_{i}=\vec{a}
$$

где $\vec{v}_{i}=\partial_{i} \vec{v}, P_{i}=\partial_{i} P, \partial_{i}-$ дифференцирование по $I_{i}$, а коэффициенты уравнения после сокращения на множитель $\mu$ могут быть выражены через инварианты.

Введем новые инвариантные скорости $\vec{w}=\mu^{-1} D \vec{I}$, оператор дифференцирования $D_{1}=w_{i} \partial_{i}$ и $\nabla_{1}=\left(\partial_{1}, \partial_{2}, \partial_{3}\right)$. 
Справедлива цепочка равенств:

$$
\begin{aligned}
D_{1} w_{k} & =\mu^{-1} D_{1}\left(I_{k t}+\vec{u} \cdot \nabla I_{k}\right)+\cdots=\mu^{-1} D_{1}\left(B \vec{v} \cdot \nabla I_{k}\right)+\cdots=\mu^{-1} D_{1} \vec{v} \cdot B^{T} \nabla I_{k}+\cdots \\
& =-\mu^{-1} w_{i} \partial_{i} \vec{v} \cdot B^{T} \nabla I_{k}+\cdots=\mu^{-2} D I_{i} \partial_{i} \vec{v} \cdot B^{T} \nabla I_{k}+\cdots \\
& =-\mu^{-2} R^{-1} n m^{-1} P_{i} B^{-1} \nabla I_{i} \cdot B^{T} \nabla I_{k}+\cdots \\
& =-\mu^{-2} R^{-1} n m^{-1} P_{i}\left(\nabla I_{i} \cdot \nabla I_{k}\right)+\cdots .
\end{aligned}
$$

Таким образом, векторное уравнение для новых инвариантных скоростей принимает вид

$$
D_{1} \vec{w}+R^{-1} C \nabla_{1} P=\vec{b},
$$

где элементы симметричной матрицы $C c_{i j}=\mu^{-2} n m^{-1}\left(\nabla I_{i} \cdot \nabla I_{j}\right)$. Скорость $\vec{u}$ через новые инвариантные скорости выражается по формулам $\vec{u}=J^{-1}\left(\mu \vec{w}-\vec{I}_{t}\right)$, где матрища $J$ имеет элементы $J_{i}^{j}=\partial I_{i} / \partial x_{j}$. Отсюда получается выражение для дивергенции $\operatorname{div} \vec{u}=\frac{\mu \partial\left(\left(J^{-1}\right)_{i}^{k} w_{k}\right)}{\partial x_{i}}+\cdots=\mu\left(J^{-1}\right)_{i}^{k} \partial_{j} w_{k} J_{j}^{i}+\cdots=\mu \partial_{j} w_{j}+\cdots=\mu \operatorname{div}_{1} \vec{w}+\cdots$

Значит, два скалярных уравнения системы (1) примут вид

$$
D_{1} R+R \operatorname{div}_{1} \vec{w}=R b_{4}, \quad D_{1} P+A \operatorname{div}_{1} \vec{w}=b_{5}
$$

Систему (6), (7) можно упростить, если иметь дело с подмоделью газовой динамики.

Теорема 2. Заменой инвариантов $\vec{I}$ система (6), (7) приводится к каноническому смешанному виду с диагональной матрицей $C_{1}$, если собственные ортонормированные векторы $\vec{e}_{i}$ первоначальной матрицы С удовлетворяют равенствам

$$
\vec{e}_{i} \cdot \operatorname{rot} \vec{e}_{i}=0, \quad i=1,2,3
$$

ДокАЗАТЕЛЬСтво. Сделаем замену инвариантов по формулам $I_{i}^{\prime}=\varphi_{i}\left(I_{1}, I_{2}, I_{3}\right)$, $i=1,2,3$, с невырожденной матрищей Якоби $M=\left(\partial \varphi_{i} / \partial I_{1}\right) ; \nabla_{1}=M^{T} \nabla_{1}^{\prime}, \vec{w}^{\prime}=M \vec{w}$. При этом выполняются соотношения $D_{1}=\vec{w} \cdot \nabla_{1}=\vec{w}^{\prime} \cdot \nabla_{1}^{\prime}=D_{1}^{\prime}, \operatorname{div}_{1} \vec{w}=\operatorname{div}_{1}^{\prime} \vec{w}^{\prime}+\cdots$, где многоточием обозначены слагаемые, не содержащие производных.

Уравнения (6), (7) после замены сохраняют свой вид в новых переменных, лишш матрица $C$ заменяется на матрицу $C_{1}=M C M^{T}$. Пусть $\lambda_{i}$ есть действительные собственные числа матрищы $C, \vec{e}_{i}\left(e_{1 i}, e_{2 i}, e_{3 i}\right), i=1,2,3,-$ ортонормированньй базис из собственных векторов. В базисе из собственных векторов с матрицей перехода $E=\left(e_{i j}\right)$ матрица $C_{1}$ примет диагональньй вид $C_{1}=\operatorname{diag}\left(\lambda_{1}, \lambda_{2}, \lambda_{3}\right)=E^{T} C E$. Если положить $M=E^{T}$, то получатся уравнения для нахождения замены инвариантов, необходимой для приведения системы к каноническому виду: $\partial \varphi_{i} / \partial I_{j}=e_{j i} ; i, j=1,2,3$. Эти системы могут быть несовместны. Диагональньй вид матрицы $C_{1}$ не изменится, если растянуть столбики матрищы $E: \vec{e}_{i} \rightarrow \mu_{i} \vec{e}_{i}$. Тогда $C_{1}=\operatorname{diag}\left(\mu_{1}^{2} \lambda_{1}, \mu_{2}^{2} \lambda_{2}, \mu_{3}^{2} \lambda_{3}\right)$, а система для определения замены примет вид

$$
\frac{\partial \varphi_{i}}{\partial I_{j}}=\mu_{i} e_{j i} ; \quad i, j=1,2,3
$$


Условия совместности системы (9) состоят из 9 уравнений для функций $\mu_{i}, i=1,2,3$. Линейная комбинация этих уравнений для каждой из функций $\mu_{i}$ приводит к условиям (8) для матрицы $C$. Остальные уравнения образуют совместную в силу (8) систему уравнений для определения $\mu_{i}, i=1,2,3$ :

$$
\begin{aligned}
& e_{1 i} \partial_{2} \mu_{i}=e_{2 i} \partial_{1} \mu_{i}-\mu_{i}\left(\partial_{2} e_{1 i}-\partial_{1} e_{2 i}\right), \\
& e_{1 i} \partial_{3} \mu_{i}=e_{3 i} \partial_{1} \mu_{i}-\mu_{i}\left(\partial_{3} e_{1 i}-\partial_{1} e_{3 i}\right) .
\end{aligned}
$$

ЗАмечАниЕ. Для одномерных подалгебр, допускаемых уравнениями газовой динамики, инвариантные подмодели таковы, что условия (8) всегда выполнены. Таким образом, канонический смешанньй вид инвариантных подмоделей задается системами вида (6) , (7) с диагональной матрищей.

\section{СПИСОК ЦИТИРОВАННОЙ ЛИТЕРАТУРЫ}

[1] Овсянников Л.В.Программа подмодели. Газовая динамика // ПММ. 1994. Т. 58. № 4. C. $30-55$.

[2] Овсянников Л. В. Групповой анализ дифференциальных уравнений. М.: Наука, 1978.

[3] Головин С. В. Оптимальная система подалгебр для алгебры Ли операторов, допускаемых уравнениями газовой динамики в случае политропного газа. Новосибирск: Институт гидродинамики, 1996.

[4] Черевко А. А. Оптимальная система подалгебр для алгебры Ли операторов, допускаемых системой уравнений газовой динамики с уравнениями состояния $p=f(S) \rho^{5 / 3}$. Новосибирск: Институт гидродинамики, 1996.

Институт механики Уфимского научного центра РАН

Поступило E-mail: habirov@imran.rb.ru 